\title{
DNA Constraints Allow Rational Control of Macromolecular Conformation
}

\author{
Chandrasekhar V. Miduturu and Scott K. Silverman* \\ Department of Chemistry, University of Illinois at Urbana-Champaign, 600 South Mathews Avenue, \\ Urbana, Illinois 61801 \\ Received March 26, 2005; E-mail: scott@scs.uiuc.edu
}

As the canonical image of molecular biology, the DNA double helix exemplifies stability and structural integrity. The persistence length of duplex DNA is several hundred base pairs ( 1 base pair $=$ $3.4 \AA=0.34 \mathrm{~nm}),{ }^{1}$ suggesting that the DNA double helix may be a useful nanoscale construction element. ${ }^{2}$ Previous efforts used DNA as a static framework for assembling other preformed objects ${ }^{3}$ or for self-assembly of DNA, ${ }^{4}$ and dynamic DNA machines have been constructed. ${ }^{5}$ However, any practical nanotechnology must have moving parts not made solely out of DNA. In this report, we describe for the first time the application of DNA to control the conformation of another macromolecule, RNA. Our efforts suggest a more complete DNA nanotechnology in which the structures of a wide variety of molecules are brought under rational control using DNA.

Figure 1A schematically illustrates the application of a covalently attached duplex DNA constraint to control macromolecular con-

A
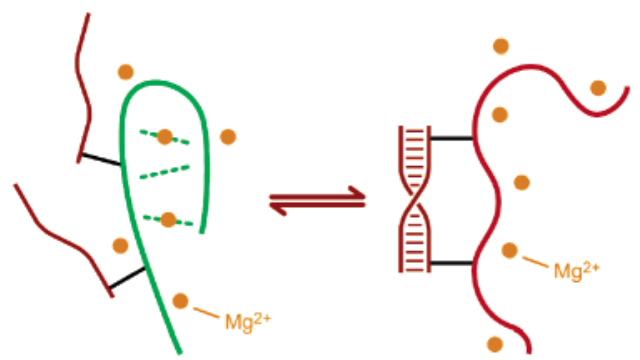

correctly folded RNA

B

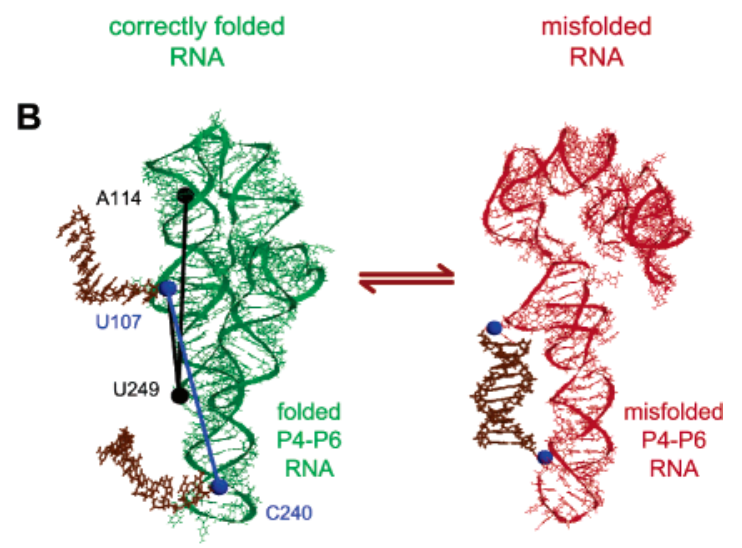

misfolded RNA
Figure 1. Controlling macromolecular RNA conformation by formation of a double-helical DNA constraint. (A) Schematic depiction of the strategy. The correctly folded RNA is stabilized by interactions (green dotted lines) that require $\mathrm{Mg}^{2+}$ ions (orange). The equilibrium is controlled by the relative stabilities of the DNA duplex and the RNA structure. The RNA stability depends on the $\mathrm{Mg}^{2+}$ concentration as well as the lengths and attachment sites of the DNA strands. (B) The strategy as depicted with 3D models. The X-ray crystal structure of P4-P6 (green) ${ }^{6}$ is shown with two DNA strands (brown) appended in arbitrary conformations at the U107 and C240 2 '-positions (blue spheres). Three pairs of sites for covalently attaching DNA strands are indicated with solid lines. One possible model of misfolded P4-P6 RNA (red) is also shown. For computer modeling, see Supporting Information. formation, as applied to RNA (for a more detailed version, see Figure S2). For the macromolecular RNA, we used the Tetrahymena group I intron P4-P6 domain (Figure 1B). This RNA folds in a $\mathrm{Mg}^{2+}$-dependent manner via formation of interactions among Watson-Crick paired and unpaired secondary structure elements. ${ }^{7}$ P4-P6 is structurally well-characterized, ${ }^{6}$ and a nondenaturing polyacrylamide gel electrophoresis (native PAGE) approach for monitoring its folding has been described. ${ }^{8}$ The two DNA strands that form the duplex constraint were attached to $\mathrm{P} 4-\mathrm{P} 6$ by reductive amination (Scheme 1). This was achieved using a 2 '-amino group

Scheme 1. Covalent Attachment of DNA to RNA ${ }^{a}$

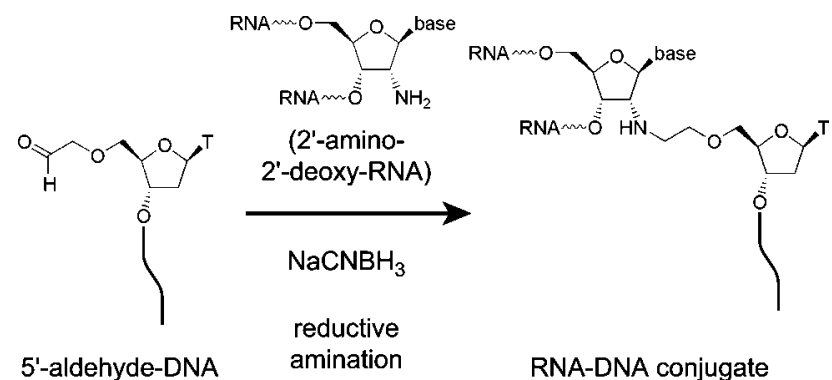

${ }^{a}$ For preparation of $5^{\prime}$-aldehyde DNA and multistep incorporation of RNA-DNA conjugates into the 160-nt P4-P6 RNA, see Figure S4.

on each of two P4-P6 nucleotides, which were coupled with complementary nonpalindromic DNA oligonucleotides that each have a $5^{\prime}$-aldehyde created by periodate oxidation. Pairs of RNA nucleotides were chosen for DNA derivatization on the basis of the P4-P6 X-ray crystal structure, ${ }^{6}$ which reveals particular 2'hydroxyl groups that are exposed to solvent (Figure 1B). Exposed 2'-hydroxyl groups are good sites for appending DNA strands without disrupting the RNA structure.

RNA generally requires $\mathrm{Mg}^{2+}$ for folding. ${ }^{9}$ When a 10-base pair (bp) DNA constraint was created using complementary DNA strands attached at P4-P6 nucleotides U107 and C240, a substantial increase in the $\mathrm{Mg}^{2+}$ requirement for $\mathrm{P} 4-\mathrm{P} 6$ folding was observed by native PAGE (Figure 2, $\boldsymbol{\Delta}$ versus $\boldsymbol{O}$ ). The increased $\mathrm{Mg}^{2+}$ requirement when the DNA constraint is attached implies a less favorable free energy of RNA folding $\left(\Delta G^{\circ}\right)$, and this effect is readily quantified from the shift in the $\mathrm{Mg}^{2+}$ midpoint of the titration curve. ${ }^{8}$ The observed energetic effect of $\Delta \Delta G^{\circ}>6 \mathrm{kcal} / \mathrm{mol}$ is consistent with the P4-P6 X-ray crystal structure. ${ }^{6}$ This structure suggests that a 10-bp duplex (length $\sim 34 \AA$ ) is too short to span U107-C240, because the $2^{\prime}$-positions are $\sim 56 \AA$ apart in the folded RNA. Therefore, the duplex DNA constraint must either fray partially, distort considerably, or melt entirely if the RNA is to fold properly (see Figure S3 for computer modeling). The $\Delta G^{\circ}$ is reduced by the energy necessary to achieve this disruption, which should cost on the order of $1-2 \mathrm{kcal} / \mathrm{mol}$ per bp. ${ }^{10}$ In contrast, when a 20 -bp DNA constraint $(\sim 68 \AA)$ was attached at the same two RNA nucleotides, almost no structural disruption was observed 


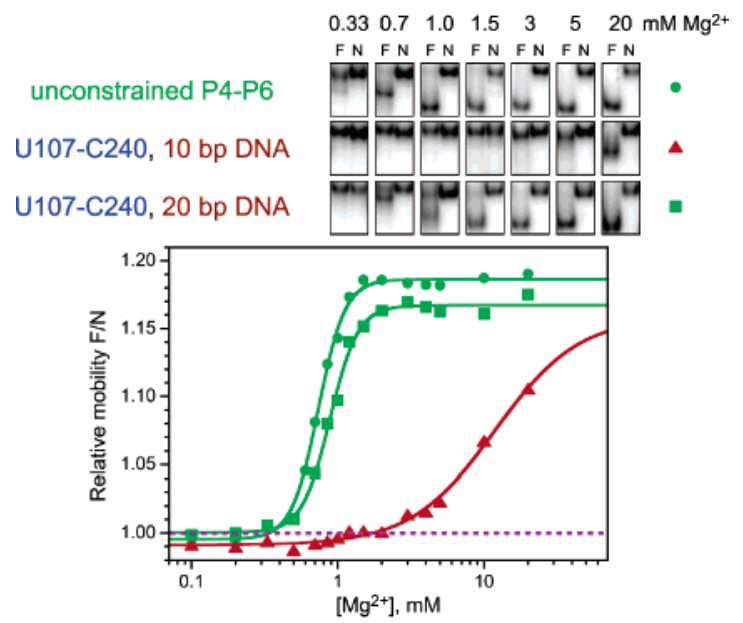

Figure 2. Nondenaturing (native) PAGE demonstrates the effects of DNA constraints on P4-P6 RNA folding. F denotes a foldable RNA sequence; $\mathrm{N}$ is nonfoldable due to nucleotide mutations that prevent a required "hinging" motion. ${ }^{7}$ The relative gel mobility of $\mathrm{N}$ is defined as 1.0 (purple dashed line). ${ }^{8}$

by native PAGE (Figure 2, $\mathbf{q}$ versus $\bullet ; \Delta G^{\circ} \approx 0.4 \mathrm{kcal} / \mathrm{mol}$ ). This is also consistent with the $\mathrm{P} 4-\mathrm{P} 6$ crystal structure and computer modeling, which suggests that a 20-bp duplex can be accommodated without distorting the RNA and with minimal fraying of the DNA (Figure S3).

Extensive control experiments demonstrated that the disruption of RNA conformation was due specifically to DNA duplex formation (Figure S5). Inverting the duplex orientation (i.e., swapping the sequences of the two attached DNA strands) led to a similar effect. In contrast, when either RNA nucleotide did not have an attached DNA strand or the two DNA strands were not complementary, RNA folding was not disrupted. When the two DNA sequences were scrambled but complementarity was retained, the RNA disruption was also maintained. This indicates that the DNA duplex itself, and not its particular sequence, controls the constraint phenomenon. Finally, covalently attaching duplex DNA to the RNA via only a single DNA terminus led to no disruption of RNA folding. This demonstrates that the mere proximity of a DNA duplex is insufficient to act as a constraint on the RNA. To establish generality, DNA strands were appended at the two other pairs of RNA nucleotides shown in Figure 1B. For both 10-bp and 20-bp constraints spanning the A114-U249 and U107-U249 positions, the observed effects were consistent with the modeling predictions (data not shown).

To provide a physically distinct assessment of RNA disruption due to the DNA constraint, dimethyl sulfate (DMS) was used to probe the P4-P6 RNA structure. ${ }^{7}$ For 10-bp DNA-constrained P4-P6, DMS probing revealed mostly unfolded RNA at low $\mathrm{Mg}^{2+}$ concentrations, whereas unconstrained P4-P6 folded normally (Figure S6). However, when the $\mathrm{Mg}^{2+}$ concentration was raised, protections characteristic of correctly folded P4-P6 were restored for the constrained RNA. Significantly, the $\mathrm{Mg}^{2+}$ dependence of the DMS protections closely paralleled the $\mathrm{Mg}^{2+}$ dependence of folding as assessed by native gels (compare Figure S6 with Figure 2 ). These data provide independent confirmation that the DNA constraint influences RNA conformation in a predictable fashion.

Here we have described a conceptually novel approach to DNA nanotechnology in which a DNA constraint actively controls the conformation of an attached RNA macromolecule. When the native $\mathrm{Mg}^{2+}$-dependent RNA conformation and the DNA constraint cannot exist simultaneously, the balance between the competing RNA and DNA structures is controlled by the $\mathrm{Mg}^{2+}$ concentration. In this study, RNA was chosen as the test system for conformational control in part because understanding RNA folding is an important area of inquiry. ${ }^{11}$ We anticipate that DNA constraints will allow detailed exploration of RNA folding landscapes, including singlemolecule studies. ${ }^{12}$ For example, misfolded RNA conformations induced by DNA constraints can now be studied directly (e.g., Figures 2 and S6), and these misfolded states may potentially be correlated with non-native RNA conformations (e.g., "kinetic traps") found in other experiments. ${ }^{13}$ Our approach should allow initiation of folding from discrete well-defined misfolded RNA states, in contrast to poorly defined "unfolded" RNA states formed by changing ionic conditions. ${ }^{14}$

Although other methods have been used to control RNA structure using triplex-forming DNA strands ${ }^{15}$ or covalent cross-links, ${ }^{16}$ our DNA constraint strategy has several advantages. (1) The DNA strands do not interact directly with the RNA, suggesting that other macromolecules such as proteins and nonbiological foldamers ${ }^{17}$ should be amenable to the DNA constraint approach. (2) Our duplex DNA constraints do not require the relatively complex doublecrossover or similar DNA motifs that have been used elsewhere. ${ }^{2}$ Instead, simple 10-20-bp DNA duplex elements are used to control the conformations of other macromolecules. (3) The noncovalent and "programmable" nature of duplex DNA formation suggests that two or more functionally orthogonal constraints should be applicable simultaneously. In other studies, covalently attached DNA strands may be useful for influencing macroscopic properties (e.g., by controlling the conformations of individual self-assembling RNA motifs). ${ }^{18}$

Acknowledgment. This research was supported by the Burroughs Wellcome Fund, the March of Dimes, the National Institutes of Health, and the UIUC Department of Chemistry. S.K.S. is a Fellow of The David and Lucile Packard Foundation. We are grateful to J. Baudry for assistance with the computer modeling experiments.

Supporting Information Available: Experimental details. This material is available free of charge via the Internet at http://pubs.acs.org.

\section{References}

(1) Hagerman, P. J. Annu. Rev. Biophys. Biophys. Chem. 1988, 17, 265.

(2) Seeman, N. C. Nature 2003, 421, 427.

(3) (a) Keren, K.; Krueger, M.; Gilad, R.; Ben-Yoseph, G.; Sivan, U.; Braun, E. Science 2002, 297, 72. (b) Yan, H.; Park, S. H.; Finkelstein, G.; Reif J. H.; LaBean, T. H. Science 2003, 301, 1882. (c) Warner, M. G.; Hutchison, J. E. Nat. Mater. 2003, 2, 272.

(4) (a) Mao, C.; Sun, W.; Seeman, N. C. Nature 1997, 386, 137. (b) Shih, W. M.; Quispe, J. D.; Joyce, G. F. Nature 2004, 427, 618

(5) (a) Mao, C.; Sun, W.; Shen, Z.; Seeman, N. C. Nature 1999, 397, 144 (b) Yurke, B.; Turberfield, A. J.; Mills, A. P., Jr.; Simmel, F. C.; Neumann, J. L. Nature 2000, 406, 605. (c) Li, J. J.; Tan, W. Nano Lett. 2002, 2 , 315. (d) Alberti, P.; Mergny, J. L. Proc. Natl. Acad. Sci. U.S.A. 2003 100, 1569. (e) Feng, L.; Park, S. H.; Reif, J. H.; Yan, H. Angew. Chem. Int. Ed. 2003, 42, 4342. (f) Liao, S.; Seeman, N. C. Science 2004, 306 Int. Ed. 2072.

(6) Cate, J. H.; Gooding, A. R.; Podell, E.; Zhou, K.; Golden, B. L.; Kundrot, C. E.; Cech, T. R.; Doudna, J. A. Science 1996, 273, 1678.

(7) Murphy, F. L.; Cech, T. R. Biochemistry 1993, 32, 5291.

(8) Silverman, S. K.; Cech, T. R. Biochemistry 1999, 38, 8691

(9) Tinoco, I., Jr.; Bustamante, C. J. Mol. Biol. 1999, 293, 271.

(10) SantaLucia, J., Jr. Proc. Natl. Acad. Sci. U.S.A. 1998, 95, 1460.

(11) Russell, R.; Herschlag, D. J. Mol. Biol. 2001, 308, 839.

(12) Zhuang, X.; Bartley, L. E.; Babcock, H. P.; Russell, R.; Ha, T.; Herschlag, D.; Chu, S. Science 2000, 288, 2048

(13) Treiber, D. K.; Williamson, J. R. Curr. Opin. Struct. Biol. 2001, 11, 309

(14) Russell, R.; Zhuang, X.; Babcock, H. P.; Millett, I. S.; Doniach, S.; Chu, S.; Herschlag, D. Proc. Natl. Acad. Sci. U.S.A. 2002, 99, 155.

(15) Moses, A. C.; Schepartz, A. J. Am. Chem. Soc. 1997, 119, 11591.

(16) (a) Allerson, C. R.; Verdine, G. L. Chem. Biol. 1995, 2, 667. (b) Glick, G. D. Biopolymers 1998, 48, 83 .

(17) Hill, D. J.; Mio, M. J.; Prince, R. B.; Hughes, T. S.; Moore, J. S. Chem Rev. 2001, 101, 3893

(18) Chworos, A.; Severcan, I.; Koyfman, A. Y.; Weinkam, P.; Oroudjev, E.; Hansma, H. G.; Jaeger, L. Science 2004, 306, 2068.

JA051950T 